\title{
SOCIABLE: creación de una plataforma para la promoción de la comunicación, rehabilitación cognitiva e interacción social en personas mayores
}

\author{
Adoración Reyes Moliner \\ Universidad Católica de Valencia San Vicente Mártir, España \\ adoracionreyes.moliner@ucv.es \\ Irene Zaragoza \\ Labhuman. Universidad Politécnica de Valencia, España \\ María Carbonell \\ Centro de día para mayores Tres Forques de Valencia, España
}

Azucena García-Palacios

Universitat Jaume I. Dpt. Psicología Básica, Clínica y Psicobiología, España

\begin{abstract}
SocIABLE (ICT-PSP n. 238891) is a project funded by the EU addressed to the population over 65 years old with different degrees of cognitive impairment. In this project technical and health care teams from four European countries participate (Norway, Italy, Greece and Spain). The main objective of SOCIABLE is to develop ICT-based tools (using Microsoft Surface and touch screen tablet PCs) to delivery cognitive rehabilitation and social activation training by means of several games. The system includes games to train different cognitive abilities (attention, memory, etc.) and a reminiscence training in order to increase social contacts among the elderly. The total number of users participating in the study is 350 (136 without cognitive impairment, 140 with mild cognitive impairment and 74 suffering dementia). A subset of the sample (278) participates in the program in the framework of the activities of the care center they attend with the support of health care staff trained in the use of SOCIABLE; a smaller amount of users (72) participates in the study from their homes. The program lenght is 12 weeks and includes 24 sessions ( 2 sessions per week). Each session lasts one hour. Participants are evaluated before using sociable. The assessment protocol includes cognitive, mood and social measures, as well as the level of expertise in the use of ICTs. After the program participants go through a post-intervention assessment and they also perform a 3-month follow-up. The preliminary results of the first group of users indicated that participants without cognitive impairment improved in some cognitive variables (MiniMental, attention, mood). In addition, the level of satisfaction with the use of ICTs was high, considering that most of the sample had no previous experience with the use of ICTs. In this paper we describe sociable and these preliminary results.
\end{abstract}

Keywords: the elderly, ICTs, cognitive rehabilitation, social activation 


\section{Resumen}

El proyecto SOCIABLE (ICT-PSP n. ${ }^{\circ}$ 238891) es un proyecto financiado por la Unión Europea, dirigido a población mayor de 65 años, con distinto grado de deterioro cognitivo. Es un proyecto en el que participan expertos técnicos y de la salud de 4 países europeos: Noruega, Italia, Grecia y España. El objetivo general de sociable es desarrollar una herramienta basada en nuevas tecnologías (mesa Surface de Microsoft y PC táctiles), en las que instalar distintos juegos (dirigidos a trabajar aspectos tales como la atención, la memoria, funciones ejecutivas, reminiscencia con el libro de la Vida, etc.) que permitan llevar a cabo rehabilitación cognitiva y que además activen las relaciones sociales entre los mayores. El número total de usuarios que participan en este estudio es de 350 (136 sin deterioro cognitivo, 140 con deterioro cognitivo leve y 74 con Alzheimer). Una parte de los usuarios (278) participa en el programa en los centros de día y residencias para mayores con la ayuda de expertos médicos y cuidadores entrenados en el uso del mismo; un número menor de usuarios (72) realiza el programa en casa. El programa tiene una duración de 12 semanas, con una frecuencia de dos sesiones semanales, de una hora de duración cada una de ellas. Los usuarios son evaluados antes de participar en el programa (se les aplica un protocolo con diferentes pruebas neuropsicológicas, se mide también el nivel de activación social y el estado de ánimo, junto al grado de familiaridad con las nuevas tecnologías entre otras variables), al finalizar el mismo y en un seguimiento a los tres meses. Los resultados preliminares del primer grupo del estudio nos indicaron que en los usuarios sin deterioro cognitivo se producía una mejoría en algunas variables cognitivas (p. ej. MiniMental, atención, mejoría de estado de ánimo). Asimismo, el nivel de satisfacción con las nuevas tecnologías era alto, teniendo en cuenta que muchos de ellos carecían de experiencia en el uso de las mismas. En este artículo presentaremos los resultados obtenidos por los dos grupos que yan han finalizado el programa.

Palabras clave: mayores, TIC, rehabilitación cognitiva, activación social

\section{Introducción}

El deterioro cognitivo y la demencia constituyen las causas más prevalentes de institucionalización, morbilidad y mortalidad en nuestros mayores. A medida que la esperanza de vida se incrementa, el número de personas aquejadas de demencia también aumenta, estimándose un crecimiento de los 24,3 millones de afectados en el 2001 a 81,1 millones en el 2040 (Alzheimer's Association, 2009). Estos datos explican el creciente interés en el desarrollo de intervenciones innovadoras y eficaces destinadas a aliviar el deterioro cognitivo y funcional asociado a la demencia (Bask y otros., 2008).

En este sentido, las tecnologías de la información y la comunicación (TICs ) pueden ayudar en esta tarea.

Sociable es un proyecto financiado por el VII Programa Marco de la Unión Europea cuyo objetivo es explorar el uso de TICs para la rehabilitación cognitiva, la mejora del estado de ánimo y la reducción del aislamiento social de personas mayores sin deterioro cognitivo, con deterioro cognitivo leve y con demencia en fase inicial. En el marco de este proyecto, se ha diseñado y desarrollado un sistema que funciona en innovadoras soluciones de interacción: tecnología Surface y Tablet PC.

El objetivo final es mejorar la calidad del cuidado que se les ofrece a nuestros mayores en centros de día y residencias y también en sus propios domicilios. Desde un punto de vista tecnológico, se ha puesto especial cuidado en realizar un diseño ergonómico adecuado al grupo de edad y a los diferentes niveles de deterioro cognitivo. Por ello se ha elegido la tecnología Surface (Microsoft Surface Platform), que proporciona una interfaz que facilita el desarrollo de numerosas soluciones multitouch, una forma de interaccionar con la tecnología de forma más natural y ergonómica. 
En este artículo describimos el sistema SOCIABLE y presentamos datos preliminares de su eficacia en la mejora del estado cognitivo, anímico y funcional de los participantes en el estudio.

\section{Descripción de SOCIABLE}

\subsection{Estructura del sistema SOCIABLE}

SocIABLE incluye dos grandes áreas:

1. Juegos de rehabilitación cognitiva con el fin de entrenar distintos procesos: atención, memoria verbal, orientación viso-espacial, etc.

2. El libro de la vida: diseñado para el entrenamiento en reminiscencia. Incluye una revisión de vida, distribuida por etapas, enfatizando los aspectos positivos y con la posibilidad de compartir recuerdos y vivencias con otros usuarios.

El aspecto innovador estriba en la utilización de elementos multimedia para la realización de actividades de rehabilitación cognitiva y de reminiscencia.

\subsection{Descripción de la aplicación del progra- ma de intervención}

El programa se puede aplicar en el centro de día o residencia mediante la mesa Microsoft Surface ${ }^{\mathrm{Tm}}$. Estas sesiones las administra un profesional del centro y pueden ser individuales o en grupo (un máximo de tres usuarios). También se puede aplicar el programa en el domicilio de los usuarios. Estas sesiones son individuales y se administran de forma autoaplicada o con la ayuda de un familiar. En las figuras 1 y 2 se ofrece una imagen de una sesión grupal y una sesión individual.

El programa incluye 24 sesiones de una hora de duración que se administran en tres meses a razón de dos sesiones por semana. El contenido de cada sesión incluye una parte de entrenamiento en juegos cognitivos y otra parte en reminiscencia con el libro de la vida. El contenido de las sesiones (p. ej. qué juegos se utilizan) lo programan los profesionales al cuidado de las personas mayores.

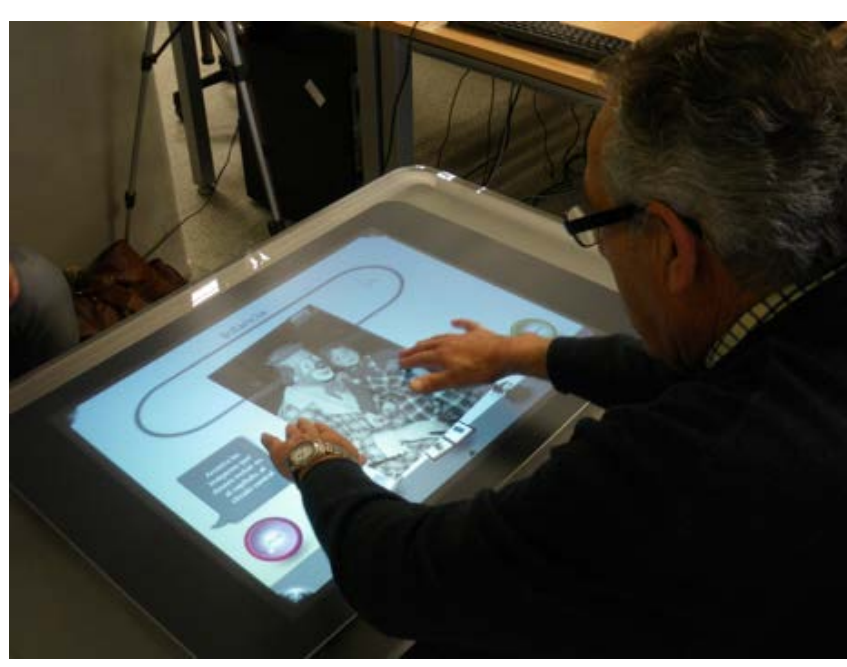

Figura 1. Imagen de un usuario en una sesión individual de reminiscencia con el libro de la vida

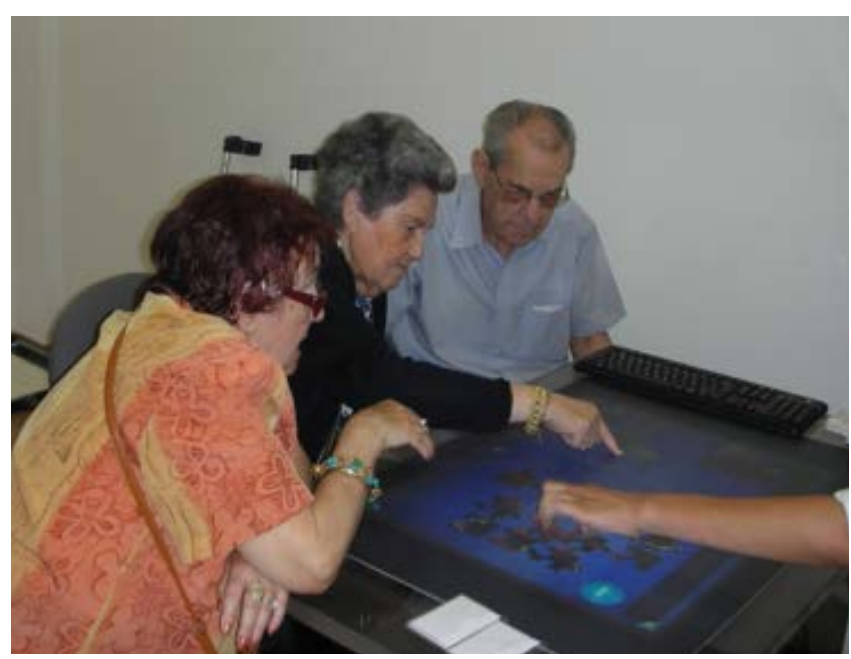

Figura 2. Imagen de una sesión de rehabilitación cognitiva en grupo

\section{Descripción del estudio empírico y resultados preliminares}

Tras el diseño y desarrollo de sociABLE, se está llevando un estudio empírico para la validación preliminar del mismo.

El número total de usuarios que participan en este estudio es de 350 (136 sin deterioro cognitivo, 140 con deterioro cognitivo leve y 74 con Alzheimer) pertenecientes a cuatro países europeos (Italia, Grecia, Noruega y España). Una parte de los usuarios (278) participa en el programa en los centros de día y residencias para mayores con la ayuda de expertos médicos y cuidadores entrenados en el 
uso del mismo; un número menor de usuarios (72) realiza el programa en casa. El programa tiene una duración de 12 semanas, con una frecuencia de dos sesiones semanales, de una hora de duración cada una de ellas.

Los usuarios son evaluados antes de participar en el programa por medio de una batería de pruebas neuropsicológicas que incluyen la valoración en las siguientes áreas: orientación, razonamiento abstracto, memoria verbal y viso-espacial, funciones ejecutivas, atención, lenguaje, estado de ánimo y estado funcional. Tras dicha evaluación son asignados de forma aleatoria a dos condiciones experimentales:

1. Condición lista de espera.

2. Condición de intervención: programa sociABLE.

Los participantes pertenecientes a la condición lista de espera son evaluados de nuevo tras tres meses. Después de esta evaluación se les ofrece la posibilidad de utilizar sociable. A los participantes asignados a la condición de intervención se les aplica el programa y son evaluados al finalizar el mismo y tres meses después con el fin de explorar los efectos a más largo plazo. En la evaluación posintervención se evalúa el grado de satisfacción de los usuarios con la intervención y el uso de TIC.

Han participado 124 personas en el estudio empírico hasta la fecha, de las cuales 102 pertenecían al grupo de población sin deterioro cognitivo, 57 al grupo de personas con deterioro cognitivo leve y 55 al grupo de personas diagnosticadas de demencia en fase inicial. De ese total, 23 participantes pertenecían a personas que utilizaban el programa desde casa y el resto a personas que lo hicieron en un centro de día o residencia. En el transcurso del ensayo se han producido 13 abandonos.

Los análisis realizados en el momento preintervención indicaron que no había diferencias en el nivel educativo y edad entre los grupos lista de espera e intervención. Se han realizado análisis preintervención y posintervención en los tres grupos por separado en función del deterioro cognitivo ( $\sin$ deterioro, deterioro leve y demencia) y se ha encontrado que se produjo una mejoría estadísticamente significativa en el deterioro cognitivo me- dido por el MiniMental en el grupo con demencia y una mejoría importante aunque sin alcanzar la significación estadística en el grupo sin deterioro cognitivo.

En el grupo de deterioro leve no se obtuvieron diferencias entre el grupo lista de espera y el grupo de intervención. Las mejorías en procesos cognitivos específicos fueron estadísticamente significativas sobre todo en el grupo sin deterioro cognitivo: memoria verbal y viso-espacial y atención. En el grupo con deterioro cognitivo leve solo se produjeron mejorías significativas en el lenguaje. Respecto al estado de ánimo y la activación social, se produjo una mejoría significativa en el grupo de deterioro cognitivo leve.

Por último, nos gustaría señalar que los participantes manifestaron un alto grado de satisfacción con el programa y con el uso de TIC. Este resultado es importante dado que la gran mayoría de los usuarios no tenían experiencia con el uso de las tecnologías.

\section{Conclusiones}

En este artículo hemos presentado una herramienta innovadora para la administración de entrenamiento en rehabilitación cognitiva, la mejora del estado de ánimo y la reducción del aislamiento social. Esta herramienta denominada SOCIABLE es fruto del trabajo conjunto de diversos equipos de investigación del área de la tecnología y de la salud de cuatro países europeos en el marco de un proyecto financiado por la UE.

El sistema socIABLE presenta una gran flexibilidad. Puede ser aplicado a distintos grupos de personas mayores con grados de deterioro cognitivo variable (desde personas sin deterioro a personas diagnosticadas de demencia). Además, puede ser utilizado de forma totalmente autoaplicada mediante un PC tablet en el domicilio del usuario o ser utilizado en sesiones grupales dirigidas por un profesional para fomentar la activación social en el marco de las actividades ofrecidas por un centro de asistencia para personas mayores.

El programa incluye entrenamiento en rehabilitación cognitiva y en actividades destinadas a mejorar el estado de ánimo y la activación social. El 
programa está estructurado en 24 sesiones de una hora de duración que se aplican en 12 semanas.

Los resultados preliminares indican que la utilización del programa, comparado con una condición lista de espera, produce mejorías en el deterioro cognitivo general y en procesos específicos. También se producen beneficios en la activación social. El grupo de personas que más se benefician es el de aquellas personas que no tienen deterioro cognitivo. Por ello, pensamos que, si estos resultados se confirman y se replican en el estudio completo y en otros estudios, SOCIABLE podría ser una buena herramienta para retrasar el deterioro cognitivo, afectivo y social que aparece asociado al envejecimiento.

Por último, nos gustaría destacar que uno de los resultados más atractivos que estamos obteniendo es que los mayores que participan en el estudio, sin tener experiencia con el uso de tecnologías (en muchas ocasiones, no han utilizado ni siquiera un teléfono móvil), han sido capaces de interactuar con el programa y han manifestado un grado de satisfacción muy elevado. Este resultado nos indica que los mayores se pueden beneficiar del uso de TIC, reduciendo la brecha que existe con otros grupos de edad.

\section{Referencias}

Alzheimer's Association. (2009). Alzheimer's Disease Facts and Figures, Alzheimer's \& Dementia, 5 (3).

Basak, C. y otros. (2008). Can training in a real-time strategy video game attenuate cognitive decline in older adults? Psychology of Aging, 23, 765-77. 